Ophthalmologe 2011 · 108:409

DOI 10.1007/s00347-010-2184-x

Online publiziert: 13. April 2011

(c) Springer-Verlag 2011

W.A. Lagrèze

Universitäts-Augenklinik Freiburg

\title{
Erkrankungen der Orbita
}

\section{Ein Randbereich der Ophthalmologie?}

Das Leitthema dieser und der folgenden Ausgabe von Der Ophthalmologe sind die Erkrankungen der Orbita. In der Artikelserie in diesem Heft steht als häufigste Orbitaerkrankung die endokrine Orbitopathie im Vordergrund, im nachfolgenden Heft sind es die Behandlung von Orbitatumoren und die Orbitarekonstruktion.

Die Orbita umgibt das Auge und beinhaltet einen Teil des Tränensystems und ein komplexes System bindegeweblicher Strukturen, welche die Augen und Lider in Position halten, schützen und bewegen. Entsprechend fallen viele Orbitaerkrankungen durch Sehstörungen und Lidveränderungen auf, so dass Betroffene zuerst einen Augenarzt oder eine Augenklinik aufsuchen.

Die Orbita kann von verschiedenen Erkrankungen betroffen sein. Diese umfassen angeborene Fehlbildungen, Verletzungen von Weichteilen und knöchernen Strukturen, Entzündungen (teils im Rahmen endokrinologischer Erkrankungen), Gefäßerkrankungen und nicht zuletzt Tumorerkrankungen, die gravierende Konsequenzen haben können und aus der Orbita in benachbarte Strukturen des Schädels einwachsen können. Entsprechend sind an der Behandlung orbitaler Erkrankungen viele Fachdisziplinen beteiligt.

Innerhalb der Augenheilkunde ist die Orbita ein Bereich, auf den sich die Aufmerksamkeit des Fachs selten konzentriert. Die Gründe dafür sind vielfältig: Im Vergleich zu anderen Augenerkrankungen sind Orbitaerkrankungen selten. In der Ausbildung wird dieser Bereich oft nur marginal abgedeckt. Ferner ist die Orbita keinem medizinischen Fachgebiet eindeutig zugeordnet. Sie wird - je nach persönlicher Erfahrung und Interesse der Kollegen und Operateure an den verschie- denen Standorten - häufig von anderen Disziplinen, die sich mit dem Kopfbereich beschäftigen, mitbetreut.

Um dem fächerübergreifenden Aspekt der Orbitaerkrankungen gerecht zu werden, haben sich in den letzten Jahren in Deutschland einige Orbitazentren formiert. An unserem Standort sind folgende Abteilungen und Schwerpunkte beteiligt: Augenheilkunde mit der Sektion Neuroophthalmologie/Kinderaugenheilkunde/ Schielbehandlung; Hals-, Nasen-, Ohrenheilkunde; Innere Medizin mit Hämatologie/Onkologie und Endokrinologie; Mund-, Kiefer- und Gesichtschirurgie; Neurochirurgie; Neuroradiologie; Plastische und Handchirurgie; Pädiatrische Hämatologie und Onkologie; Strahlenheilkunde. Deren Vertreter treffen sich ein$\mathrm{mal}$ in der Woche zu einer gemeinsamen Konferenz, bei welcher Fälle interdisziplinär diskutiert und verbindliche Behandlungspläne festgelegt werden.

Schonendere Operationstechniken sowie Fortschritte in der radiologischen Bildgebung und Bestrahlungsplanung haben in den letzten Jahren neue und bessere Behandlungsmöglichkeiten eröffnet, welche Ihnen die Autoren dieses Leitthemas nahebringen wollen. An dieser Stelle möchte ich allen Autoren und Mitgliedern des Freiburger Orbitazentrums sehr herzlich für ihr Engagement und ihre Beiträge danken.

Ihr

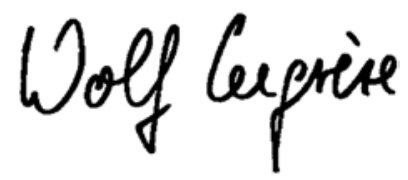

Prof. Dr. Wolf A. Lagrèze

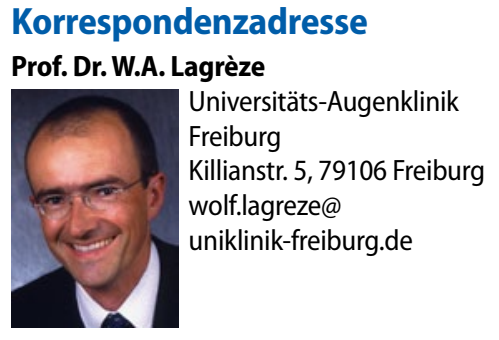

